\title{
Complete occlusion of the left main coronary artery ostium in Takayasu arteritis
}

\author{
Abbas Emami Nia, MD, and Ahmad Ali Amirghofran, MD, Shiraz, Iran
}

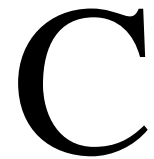

oronary artery involvement occurs in about $10 \%$ of patients with Takayasu arteritis (TA), with the coronary ostia involved most frequently. We report the rare case of a patient with complete obliteration of the left coronary ostium found intraoperatively. This has not previously been reported in the literature. The patient also had a ventricular septal defect (VSD) and severe aortic regurgitation. She underwent on-pump coronary artery bypass grafting (CABG), aortic valve replacement, and VSD closure in a single operative session.

\section{Clinical Summary}

A 27-year-old woman was admitted to our facility because of exertional angina. She had had TA diagnosed at the age of 24 years. Her blood pressure readings were $95 / 65 \mathrm{~mm} \mathrm{Hg}$ in the right arm and 120/ $70 \mathrm{~mm} \mathrm{Hg}$ in the left arm. Results of head, neck, ear, and eye examinations were unremarkable, and no bruits were heard over the subclavian and common carotid arteries. Cardiac examination revealed a grade III/VI blowing murmur best heard at the right sternal border. Absence of right radial and brachial pulses was evident. The common carotid artery pulses were diminished. Laboratory values were within normal ranges, except for high erythrocyte sedimentation rate $(60 \mathrm{~mm} / \mathrm{h})$ and C-reactive protein $(2 \mathrm{mg} / \mathrm{dL})$.

The electrocardiogram was remarkable for ST-segment elevation and symmetric $\mathrm{T}$ inversion in leads $\mathrm{I}, \mathrm{aVL}, \mathrm{V}_{1}$, and $\mathrm{V}_{2}$. Echocardiography showed normal left ventricular function with a $70 \%$ ejection fraction, a perimembranous subaortic VSD and severe aortic regurgitation. Coronary angiography revealed the right coronary artery to be normal, but the left coronary ostium could not be found (Figure 1). The left coronary system had faint retrograde filling from the right coronary artery. The patient was scheduled for emergency CABG and aortic valve replacement.

During the operation, we found that the left coronary ostium was totally occluded. The left sinus of Valsalva was flat and covered with normal-looking intima. A small, shallow depression was noticed at the normal site of left coronary ostium (Figure 2). The root of aorta was dilated, and the aortic wall was thickened.

The aortic valve was replaced, and the VSD was repaired. Onpump CABG was performed with a free right internal thoracic graft

From the Division of Cardiac Surgery, Department of Surgery, Shiraz University of Medical Sciences, Faghihi Hospital, Shiraz, Iran.

Received for publication Sept 29, 2007; accepted for publication Nov 26, 2007.

Address for reprints: Abbas Emami Nia, MD, Division of Cardiovascular Surgery, Department of Surgery, Shiraz University of Medical Sciences, Faghihi Hospital, Shiraz, Iran 71357 (E-mail: emaminia@gmail.com).

J Thorac Cardiovasc Surg 2008;135:695-6

$0022-5223 / \$ 34.00$

Copyright (C) 2008 by The American Association for Thoracic Surgery doi:10.1016/j.jtcvs.2007.11.031

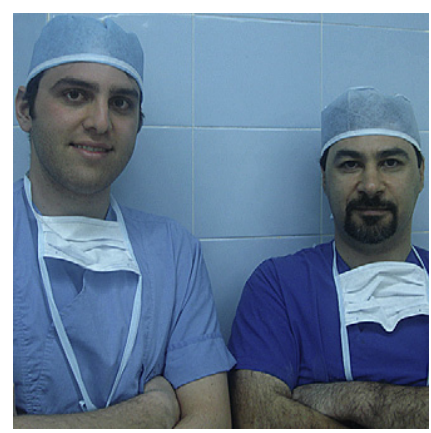

Drs Nia and Amirghofran (left to right)

to the left anterior descending coronary artery and a saphenous vein graft to the first obtuse marginal artery. There were no postoperative cardiac or noncardiac complications. At 1 year of follow-up, the patient was doing well, without any signs of new cardiac ischemia.

\section{Discussion}

When coronary arteries are involved in TA, their proximal, rather than distal, segments are more often affected. The reason for this ostial involvement is extension of the inflammation-induced intimal proliferation and fibrous contraction from the ascending aorta to the coronary ostia. Many reports exist on cases with as much as 98\% left main trunk stenosis. To the best of our knowledge, however, occlusion of the left coronary ostium as a result of progressive inflammation has not been previously reported.

Although CABG is the procedure with the best long-term patency rate, ${ }^{1}$ it may be difficult in TA patients. Inflammation is persistent in more than $40 \%$ of cases even after treatment, ${ }^{2}$ and this would jeopardize the insertion sites of new grafts. In addition, a calcified aorta makes insertion of the aortic cannulas difficult.

A major issue in patients with TA undergoing CABG is restenosis of the grafts. Although internal thoracic artery graft patency is significantly higher than that of vein graft, it should not be used in

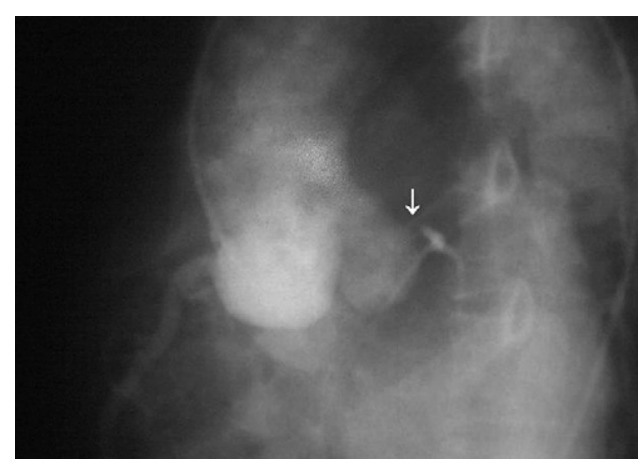

Figure 1. Coronary angiogram. Note discontinuity of left main coronary artery (arrow) from its origin. 


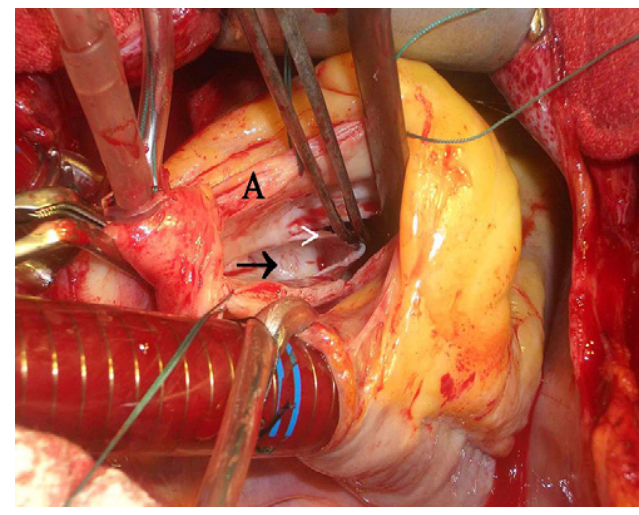

Figure 2. Intraoperative image of left coronary sinus. Total obliteration of left coronary ostium is seen (black arrow). Also note thickened aortic wall (A). White arrowhead indicates left coronary cusp of aortic valve.

patients with TA because of the high rate of subclavian artery occlusion. The long-term graft patency of saphenous vein grafts is reported to be about $60 \%$ at 4 years. $^{3}$

Percutaneous coronary intervention is the choice of revascularization for poor surgical candidates with calcified aortas. Technical and equipment advances have enabled stenting in the unprotected left main coronary artery to be performed with a high initial success rate and acceptable short- to intermediate-term outcomes. Favorable long-term outcomes have also been reported for patients with good left ventricular function, although restenosis occurs in $20 \%$ of patients. ${ }^{2}$ For patients with previously inserted multiple bare metal stents and restenosis, Furukawa and colleagues ${ }^{2}$ used sirolimuseluting stents and observed good 6-month outcomes.

In conclusion, we report a case of TA with obliteration of the left coronary ostium. This was successfully treated with CABG, and the patient was event free at 1 year. CABG, although difficult in some cases of TA, remains the best revascularization option for patients with significant left main trunk stenosis.

We thank Dr Kenneth J. Cherry, University of Virginia, and Sai Jahann for their editing of the manuscript.

\section{References}

1. Liang P, Hoffman GS. Advances in the medical and surgical treatment of Takayasu arteritis. Curr Opin Rheumatol. 2005;17:16-24.

2. Kerr GS, Hallahan CW, Giordano J, Leavitt RY, Fauci AS, Rottem M, et al. Takayasu arteritis. Ann Intern Med. 1994;120:919-29.

3. Ando M, Sasako Y, Okita Y, Tagusari O, Kitamura S, Matsuo H. Surgical considerations of occlusive lesions associated with Takayasu's arteritis. Jpn Heart J. 2000;48:173-9.

\title{
Anomalous left coronary artery from the pulmonary artery with an intramural course within the aortic wall: Report of 3 surgical cases
}

\author{
Steven P. Goldberg, MD, ${ }^{a}$ Max B. Mitchell, MD, ${ }^{a}$ David N. Campbell, MD, ${ }^{a}$ Cecile Tissot, MD, ${ }^{b}$ and \\ Francois Lacour-Gayet, MD, ${ }^{a}$ Denver, Colo
}

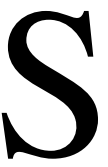

ince 1996, we have operated on 15 patients with anomalous left coronary artery from the pulmonary artery (ALCAPA). Three cases involved a rare variant in which the left coronary artery (LCA) also took an intramural aortic course in the left sinus of Valsalva (LSV) and then exited the aorta to assume its usual pattern.

\footnotetext{
From the Divisions of Cardiothoracic Surgery ${ }^{\mathrm{a}}$ and Pediatric Cardiology, ${ }^{\mathrm{b}}$ The Children's Hospital, Denver, Colo.

Received for publication Sept 27, 2007; accepted for publication Oct 15, 2007.

Address for reprints: Max B. Mitchell, MD, The Children's Hospital, Denver, Division of Cardiothoracic Surgery, 13123 East 16th Avenue, Box B200, Aurora, Colorado 80045 (E-mail: Mitchell.Max@tchden.org).

J Thorac Cardiovasc Surg 2008;135:696-8

$0022-5223 / \$ 34.00$

Copyright $\odot 2008$ by The American Association for Thoracic Surgery doi:10.1016/j.jtcvs.2007.10.033
}

\section{Clinical Summary}

PATIENT 1. A 3-month-old infant underwent an emergency operation for ALCAPA. The LSV was unusually adherent to the adjacent pulmonary artery. The LCA originated from the inferior proximal right pulmonary artery and then took an intramural course in the LSV with normal distal branching. Inside the aorta a "dimple" was observed at the usual site of the LCA origin. A button was transferred to the aorta. Transesophageal echocardiographic analysis demonstrated antegrade LCA flow on cardiopulmonary bypass that was not demonstrable after weaning. The transferred coronary artery was revised by opening the ostium into the distal part of the intramural segment. The opened proximal LCA, adjacent aortic wall, and intima of the unroofed LCA were approximated with interrupted 8-0 Prolene sutures. A left ventricular assist device was required and was weaned at 48 hours. Delayed sternal closure was then achieved. Echocardiographic analysis 6 years after the operation demonstrated a widely patent LCA and normal ventricular function.

PATIENT 2. A 3-year-old patient underwent nonemergency repair of ALCAPA. The LCA originated from the inferior proximal right pulmonary artery. There was dense apposition of the pulmonary artery and adjacent LSV. Probing confirmed an intramural course 